REVIEW ARTICLE

\title{
Star Fruit Intoxication
}

\author{
*N Mahmood ${ }^{1}$ \\ ${ }^{1}$ Dr. Nazneen Mahmood, Associate Professor \& Head of Nephrology, AKMMCH \\ *Corresponding Author \\ Date of submission: 28 December 2014 Date of acceptance: 03 March 2015
}

\begin{abstract}
Now a day Acute Kidney Injury and Chronic Kidney Disease are the two common problems worldwide with increased mortality and morbidity as well as health care cost. Nephrotoxicity and neurotoxicity by the Star fruit, sometimes fatal, is far more frequent than reported. Star fruit belongs to the Oxalidaceae family, species Averrhoea carambola, is a popular fruit among Orientals. The aim of the study is to create awareness among the population regarding star fruit intoxication, to reduce the development of Acute Kidney Injury in general population, to reduce the mortality and burden of morbidity from chronic renal failure, to reduce the mortality of the patients of End stage renal disease taking regular haemodialysis and to reduce the mortality and burden of morbidity of the people suffering from Diabetes mellitus and Hypertension. Star fruit juice produces acute renal injury not only through the obstructive effect of calcium oxalate crystals, but also inducing apoptosis of renal epithelial cells, which may be caused by the levels of oxalate in the fruit. There have been reports of hiccup, confusion and occasional fatal outcomes in uremic patients after ingestion of star fruit. An excitatory neurotoxin from star fruit has been implicated although the exact nature of this toxic substance has not been identified. High quantities of oxalate in empty stomach and dehydrated state may pose an additional risk for development of renal injury. To avoid acute oxalate nephropathy, pure sour carambola juice or mild diluted post pickled juice should not be consumed in large amounts, especially in an empty stomach and dehydrated state. It is recommended that uremic patients should totally abstain from star fruit due the rare but potentially fatal complications.
\end{abstract}

Key Words: Star fruit, Nephrotoxicity, Neurotoxicity, Oxalidaceae

\section{Introduction}

Now a day Acute Kidney Injury and Chronic Kidney Disease are the two common problems worldwide with increased mortality and morbidity as well as health care cost. Nephrotoxicity and neurotoxicity by the Star fruit, sometimes fatal, is far more frequent than reported ${ }^{2}$.

Star fruit (Figure: 1,2) belongs to the Oxalidaceae family, species Averrhoea carambola, is a popular fruit among Orientals ${ }^{1}$. Star fruit is one of the plant sources that contain highest concentration of oxalic acid; $100 \mathrm{~g}$ of fresh fruit contains 50,000-95,800 ppm of oxalic $\mathrm{acid}^{2}$. Sour carambola juice is a popular beverage in Taiwan. Commercial carambola juice usually is prepared by pickling and dilution processes that reduce oxalate content markedly ${ }^{2}$.

There have been reports of hiccup, confusion and occasional fatal outcomes in uremic patients after ingestion of star fruit. An excitatory neurotoxin from star fruit has been implicated although the exact nature of this toxic substance has not been identified ${ }^{1}$.

\section{Aims and Objectives}

General

To create awareness among the population regarding star fruit intoxication.

\section{Specific}

1. To reduce the development of Acute Kidney Injury in general population.

2. To reduce the mortality and burden of morbidity from Chronic renal failure.

3. To reduce the mortality of the patients of End stage renal disease taking regular Haemodialysis.

4. To reduce the mortality and burden of morbidity of the people suffering from Diabetes mellitus and Hypertension. 


\section{Literature Review}

Mechanism of Star fruit induced Acute Renal Failure:

To investigate the mechanisms of star fruit associated acute oxalate nephropathy, the nephrotoxic effect of star fruit was examined in both cellular experiments and animal models. Star fruit juice produces acute renal injury not only through the obstructive effect of calcium oxalate crystals, but also inducing apoptosis of renal epithelial cells, which may be caused by the levels of oxalate in the fruit $3,4,5$.

\section{Renal Biopsy findings}

Renal biopsies (Figure: 3) indicated a typical histological appearance of acute tubular necrosis and oxalate nephropathy. Light microscopy showed oxalate crystals (arrows) within the lumina and tubular walls surrounded by inflammatory infiltration that included lymphocytes, histiocytes and primarily neutrophils. Tubular focal dilation, desquamated cells in the lumina, lining epithelial cell flattening and interstitial oedema were also present. Renal sections were analysed under polarized light and showed colourless oxalate crystals with a pattern of birefringence within the tubular lumina. The oxalate crystals displayed a typical black colour after Von Kossa staining. Light microscopy showing oxalate crystals (arrows) within the lumina and tubular walls surrounded by inflammatory infiltration (Masson's Trichrome, original magnification $\times 40$ ). Renal section analysed under polarized light showing several colourless oxalate crystals with a pattern of birefringence within the tubular lumina (Haematoxylin and eosin, original magnification $\times 10$ ). Renal section stained after Von Kossa stain displaying oxalate crystals with a typical black colour (original magnification $\times 10)^{2}$.

\section{Sources of Oxalic acid}

Buckwheat, Star fruit, Black pepper, Parsley, Poppy seeds, Rubarb stalks, Amaranth, Spinach, Chard, Beets, Cocoa, Chocolate, Most Nuts, Most Berries, Beans ${ }^{6}$.

\section{Mechanism of Star fruit induced Status Epilepticus:}

Some researchers suspect that there are different toxins in different star fruit subspecies, as well as powerful neurotoxin that can accumulate in blood and cross the blood-brain-barrier in patients with chronic renal disease, eventually causing refractory status epilepticus ${ }^{7,8}$. Single voxel protein and 31 phosphorus MR spectroscopic studies revealed elevation of lactate, elevation of inorganic phosphate and reduced phosphocreatinine and nucleoside triphosphates in the cerebral cortex in an intoxicated patient ${ }^{9}$. The metabolic changes indicated that neurotoxin could interfare with oxydative phosphorylation and cause energy deprivation. Although the exact nature of star fruit neurotoxin remains obscure, oxalate being abundant in star fruit, has been proposed as a possible candidate for acute nephropathy and neurotoxicity 2 . More recently, a neurotoxic fraction was obtained from star fruit, which is different from oxalic acid in chemical characterization ${ }^{10}$. The mechanism of convulsive activity is believed to specifically act on GABA ergic glutamatergic transmission system ${ }^{10}$.

\section{Effects of Star fruit on Kidney}

- Acute kidney injury from acute oxalate nephropathy and acute tubular necrosis.

- Renal stone disease.

- Deterioration of renal function in patients of Chronic kidney disease ${ }^{2,7}$.

\section{Neurological symptoms of Star fruit intoxication:}

Hiccups, Vomiting, Insomnia, Psychomotor agitation, Numbness and paresthesias of the limbs, Decrease muscle power, Mild mental confusion, Severe mental confusion progressing to coma, Seizures progressing to status epilepticus, Hemodynamic instability progressing to hypotension and shock ${ }^{7,11}$.

\section{Treatment}

Haemodialysis, especially on daily basis, is the ideal treatment for star fruit intoxication. In severe cases, continuous methods of replacement therapy may provide a superior initial procedure, since rebound effects are a common event ${ }^{7}$. Peritoneal dialysis is of no use as a treatment, especially when conciousness disorder ensue ${ }^{7}$. Sustained low efficiency daily diafiltration with hemoperfussion as a therapy for severe star fruit intoxication ${ }^{7}$.

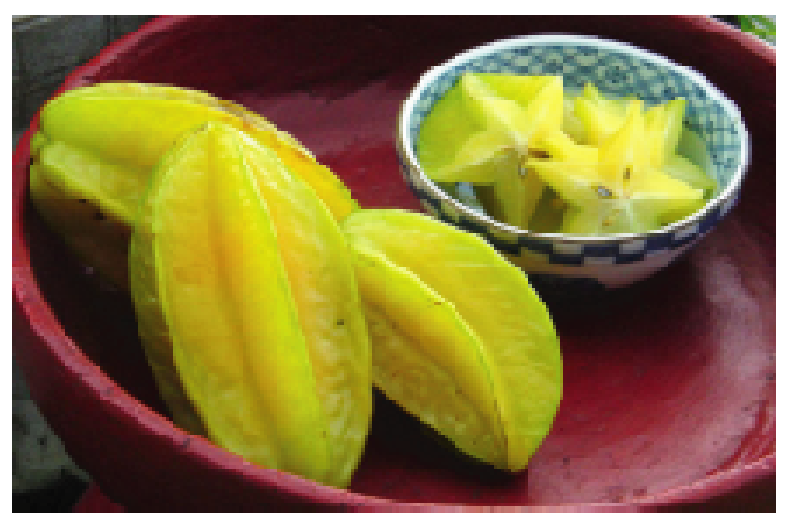

Fig1: Star fruit 


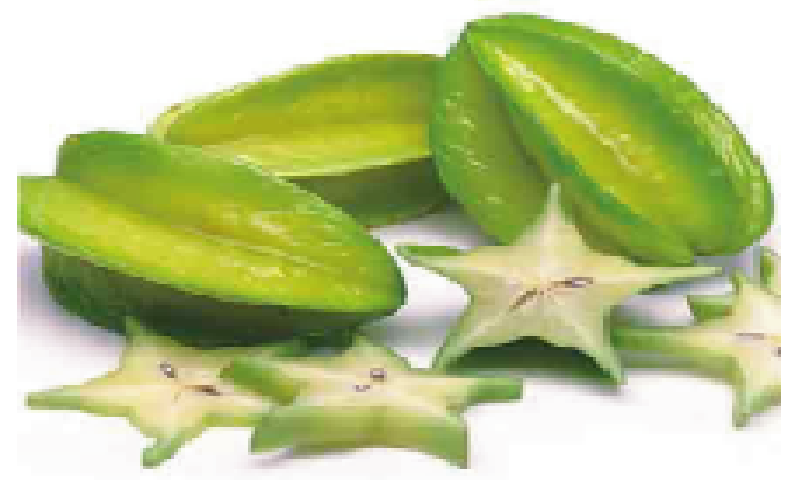

Fig 2: Kamranga

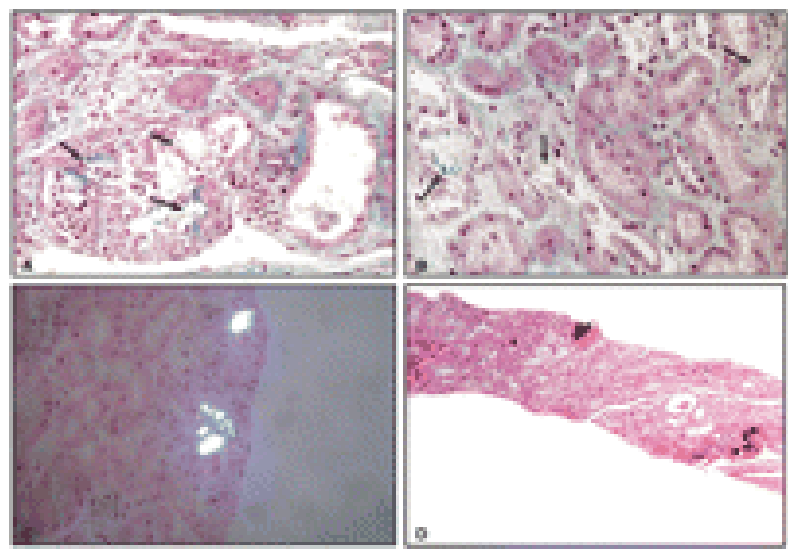

Fig 3: Slide of Renal Biopsy after Star Fruit intoxication (Acute Tubular Necrosis)

\section{Discussion}

Many studies from different countries of the world had done on star fruit intoxication. Star fruit is commonly found in tropical countries and is popular fruit in Brazil ${ }^{11}$. In 1993, Martin et al were the first to report clinical observations on the intake of this fruit11. The authors described an outbreak of intractable hiccups in patients with chronic kidney disease(CKD) on haemodialysis ${ }^{11}$.

In a study, Neto MM, Robl F, Netto JC, 1998 showed that six patients in a dialysis programme who were apparently intoxicated by ingestion of star fruit. After ingestion of 2-3 fruits or $150-200 \mathrm{ml}$ of the fruit juice, the six patients who had previously been stable in a regular dialysis programme, developed a variety of symptoms ranging from insomnia and hiccups to agitation, mental confusion and (in one case) death ${ }^{12}$. Preliminary investigations to characterize the hypothetical hypothetical neurotoxin in the fruit, an extract, when injected intraperitoneally or intra-cerebroventricularly in rats,

provoked persist convulsions of the tonic-clonic type. It appears that star fruit contains an excitatory neurotoxin ${ }^{12}$. Other reports have since confirmed these changes in CKD patients on and off dialysis ${ }^{7,13}$. Reviews on topic available in the literature have comprised years of observations and several case reports.

Tse KC et al, in 2003, showed in their review article that a group of seven patients from the dialysis center of Queen Mary and Tung Wah Hospitals who developed symptoms including hiccup, confusion, vomiting, impaired conciousness, muscle twitching and hyperkalaemia shortly after ingestion of star fruit ${ }^{1}$. Symptoms of most patients resolved after intensified dialysis or spontaneously and no mortality was observed1.

Neto MM et al, in 2003, showed in their study, that out of 32 uremic patients, 30(93.75\%) patients developed persistant and intractable hiccups, vomiting in 22(68.7\%), variable degrees of disturbed conciousness (mental confusion, psychomotor agitation) in 21(65.6\%), decreased muscle power, limb numbness, paresis, insomnia and paresthesia in $13(40.6 \%)$ and seizure in 7(21.8\%). Patients who were promptly treated with haemodialysis, including those with severe intoxication, recovered without sequelae. Patients with severe intoxication who were not treated or treated with peritoneal dialysis did not survive ${ }^{7}$.

Signata A et al, 2009, reported two cases with unknown chronic renal insufficiency, who developed severe encephalopathy after ingestion of star fruit. Diffusion-weighted MR imaging showed cortical and thalamic hyper-intense lesions related to epileptic status. They improved after being submitted to continuous hemofiltration which constitutes the most effective treatment in acute phase ${ }^{14}$.

In a study in Chang Gung Memorial Hospital, Taiwan, in 2005, Tsai MH et al showed that two patients with chronic renal failure at a pre-dialyzed stage who developed refractory status epilepticus after ingestion of star fruit ${ }^{15}$. In addition, they review 51 cases in the literature. Among 51 patients, 16 patients presented with epileptic seizures $(30 \%)$. The mortality rate was as high as $75 \%$ in patients with seizures. On the other hand in patients without seizures the mortality rate was only $0.03 \%$. Thy proposed that epileptic seizure is significantly associated with poor prognosis and that status epilepticus is an unpredictable and potentially fatal complication in star fruit intoxication ${ }^{15}$. 
Clen CL et al, 2001, showed in their study that two of the patients developed nausea, vomiting, abdominal pain and backache within hours of ingestion of large quantities of sour carambola juice, then acute renal failure followed. Pathological examinations showed typical changes of acute oxalate nephropathy ${ }^{2}$.

Niticharoenpong K et al, 2006 showed in their case report, a patient with underlying chronic kidney disease, who developed a rapid increase in serum creatinine and oxalate nephropathy after chronic ingestion of star fruit juice without overt neurotoxicity. The decline in renal function was not fully reversible after stoppage of intake ${ }^{16}$.

\section{Conclusion}

High quantities of oxalate in empty stomach and dehydrated state may pose an additional risk for development of renal injury. To avoid acute oxalate nephropathy, pure sour carambola juice or mild diluted post pickled juice should not be consumed in large amounts, especially in an empty stomach and dehydrated state. People suffering from Hypertension, Diabetes mellitus and obesity, who are at risk of kidney disease or those who have a family history of kidney disease should preferably avoid Kamranga as a precautionary measure. Patients with CKD in any stage or on dialysis should avoid eating star fruit.

\section{Recommendation}

It is recommended that uremic patients should totally abstain from star fruit due the rare but potentially fatal complications. Most of the study regarding star fruit intoxication is from other countries of the world. There is no such study among uremic patients in our country. More study as well publicity is required to create awareness of taking star fruit.

\section{Acknowledgement}

Dr. Md. Mahmudur Rahman Siddiqui, Assistant Professor, Department of Medicine, Anwer Khan Modern Medical College Hospital.

\section{Conflict of Interest}

The author has no conflict of interest to anybody.

\section{References}

1. Tse KC, Yip PS, Lam MF, et al. Star fruit intoxication in uremic patients: Case series and review of the literature. Intern Med J 2003, Jul; 33(7): 314-6.

2. Chen CL, Fang HC, Chou KJ, et al. Acute oxalate nephropathy after ingestion of star fruit. Am J Kidney Dis 2001; 37(2): 418-422.

3. Fang HC, Lee PT, Lu PJ, et al. Mechanisms of star fruitinduced acute renal failure. Food Chem Toxicol 2008; 46(5): 1744-1752.

4. Neto MM, Silva GEB, Costa RS et al. Star fruit : Simultaneous neurotoxic and nephrotoxic effects in people with previously normal renal function. NDT plus 2009; 2: 485-88.

5. Kian-Guan Lee. Star fruit intoxication with acute kidney injury. Clin Medicine. rcp journal.org 2012; 12(5): 454.

6. Sanz P, Reig R. Clinical and pathological findings in fatal plant oxalosis. A review. Am J Forensic Med Pathol 1992; 13: 342-345.

7. Neto MM, Da Costa JA, Garcia-Cairasco N, et al. Intoxication by star fruit (Averrhoa carambola) in 32 uraemic patients: treatment and outcome. Nephrol Dial Transplant 2003; 18: 120-125.

8. HJ Yap, YC Chen, JT Fang, CC Hunng. Star fruit: a neglected but serious fruit intoxication in chronic renal failure. Dial Transplant 2002; 31: 564-567.

9. YL Chan, HK Ng, CB Leung, et al. 31Phosphorus and single voxel proton MR spectroscopy and diffusion weighted imaging in a case of star fruit poisoning. AJNR Am J Neuroradiol 2002; 23: 1557-60.

10. ROG Carolino, RO Beleboni, AB Pizzo, et al. Convulsant activity and neurochemical alterations induced by a fraction obtained from fruit Averrhoa carambola (Oxalidaceae:Geraniales) Neurochem Int 2005: 46: 523-531.

11. Martin LC, Caramori JST, Barreti P, et al. Soluco intravel desencadeado por ingestao de carambola ("Averrhoa carambola") em portadores de insuficiencia renal cronica. J Bras Nephrol 1993: 15: 92-4.

12. Neto MM, Robl F, Netto JC. Intoxication by star fruit (Averrhoa carambola) in six dialysis patients? (Preliminary report). Nephrol Dial Transplant 1998; 13: 570-572.

13. Chang JM, Hwang SJ, Kuo HT, et al. Fatal outcome after ingestion of star fruit (Averrhoa carambola) in uremic patients. Am J Kidney Dis 2000; 35: 189-193.

14. Signate A, Olindo S, Chausson N, Cassinoto C, Edimo Nana M, Cabre P, Smadja D. Star fruit (Averrhoa carambola) toxic encephalopathy. Rev Neurol(Paris), 2009 Mar; 165(3): 268-72.

15. Tsai MH, Chang WN, Lui CC, et al. Status epilepticus induced by star fruit intoxication in patients with chronic renal disease. Seizure 2005 Oct; 14(7): 521-5.

16. Niticharoenpong K, Chalermsanyakorn P, Panvichian R, et al. Acute deterioration of renal function induced by star fruit ingestion in a patient with chronic kidney disease. J Nephrol 2006; 19: 682-686. 